\title{
Biexcitons in Coupled Quantum Dots as a Source for Entangled Photons
}

\author{
Oliver Gywat, Guido Burkard, and Daniel Loss \\ Department of Physics and Astronomy, Klingelbergstrasse 82, CH-4056 Basel, Switzerland
}

\begin{abstract}
We study biexcitonic states in two tunnel-coupled semiconductor quantum dots and show that such systems provide the possibility to produce polarization-entangled photons or spin-entangled electrons that are spatially separated at production. We distinguish between the various spin configurations and calculate the low-energy biexciton spectrum using the Heitler-London approximation as a function of magnetic and electric fields. The oscillator strengths for the biexciton recombination involving the sequential emission of two photons are calculated. The entanglement of the polarizations resulting from the spin configuration in the biexciton states is quantified as a function of the photon emission angles.
\end{abstract}

PACS numbers: 78.67.-Hc, 73.21.La, 71.35.-y, 72.25.Fe

Entanglement has been identified as an essential resource for many applications in the recently developed field of quantum communication and quantum computation [1]. Several quantum communication schemes have already been successfully implemented with pairs of polarization-entangled photons produced by parametric down-conversion 11, e.g. the faithful transmission of a quantum state (quantum teleportation), entanglementassisted classical communication (e.g., quantum superdense coding), and the production of a secure cryptographic key (quantum key distribution). Recently, there has also been growing interest in solid-state implementations of quantum computation using the electron spin as the qubit [2], as well as quantum communication with spin-entangled electrons. Superconductor-normal junctions in combination with quantum dots (QDs) have been suggested as a device for producing entangled electrons [3]. Still, the efficient and deterministic production of both entangled photons and electrons poses a theoretical and experimental challenge. In the case of photons, the use of electron-hole recombination in a single QD was recently suggested [4, 0 . Non-resonant excitation of a QD is expected to produce pairs of entangled photons with an efficiency (production rate/pump rate) that is about four orders of magnitude bigger than for parametric down-conversion [5].

In this paper, we study the production of polarizationentangled photons, or, alternatively, spin-entangled electrons, using the biexcitonic ground state in two tunnelcoupled QDs. For this purpose we study the low-energy biexcitonic states in coupled QDs, determining their energy spectrum and their optical properties. We concentrate on the spin configuration of the calculated states, being related to the orbital wavefunction via the Fermi statistics which is implemented in a Heitler-London (HL) ansatz for electrons and for holes. As a special quality of a double dot, we find that in the (spin-entangled) biexcitonic ground state, the biexciton favors a configuration with each QD occupied by one exciton, thus providing a basis for the separation of the entangled particles. Even though coupled QDs are usually separated by a distance less than the wavelength of the emitted light, it might still be possible to directly detect the photons at separate lo- cations. It can e.g. be expected that due to anisotropies the two dots have different preferred emission directions inclosing a fixed angle. Two subsequent photons, which are emitted with a time delay given by the exciton lifetime, could then be detected separately in the far field.

In contrast to our calculations, earlier studies for quantum computation or entanglement production with excitons in QDs concentrate on single QDs [4, 5, 6, 7, 8] and/or on charge degrees of freedom (neglecting spin) 6, 7, 8, 9, 10, 11]. Also, instead of a pure electrostatic interdot coupling [9, 11, we take into account the tunneling of electrons and holes between the coupled QDs.

Biexcitons consist of two bound excitons which themselves are formed by a conduction-band electron and a valence-band hole in a semiconductor, bound together by the attractive Coulomb interaction. Following the theory of excitonic absorption in single QDs [12], the biexcitonic states in single QDs have been investigated [13, 14, 15, 16, 17, 18, 19, 20] and single excitons in coupled QDs have been observed in experiment 10, 21]. Recently, spin spectroscopy of excitons in QDs was performed using polarization-resolved magnetophotoluminenscence [22]. Two regimes can be distinguished in the discussion of excitons confined in QDs [12. In the weak confinement limit $a_{X} \ll a_{e}, a_{h}$, where $a_{X}$ is the radius of the free exciton and $a_{e}, a_{h}$ the electron and hole effective Bohr radii in the QD, an exciton can (as in the bulk material) be considered as a boson in an external confinement potential. In the case of strong confinement $a_{X} \gg a_{e}, a_{h}$, electrons and holes are separately confined in the $\mathrm{QD}$ and the bosonic nature of the electron-hole pair breaks down. Since, e.g., in bulk GaAs $a_{X} \approx 10 \mathrm{~nm}$, we are in an intermediate regime $a_{X} \approx a_{e}, a_{h}$ for typical QD radii. Here, we start from a strong confinement ansatz, i.e. from independent electrons and holes (two of each species), and then use the HL approximation to include the Coulomb interaction and the tunneling. Unlike for bulk biexcitons, where the HL approximation fails for some values of $\xi=m_{e} / m_{h}$ [23], we are here in a different situation - much more similar to the $\mathrm{H}_{2}$ moleculebecause the single particle orbitals are defined by the strong QD confinement, the latter playing the role of the ("infinitely" heavy) protons of the $\mathrm{H}_{2}$ molecule. 
We obtain the low-energy (spin-resolved) biexciton spectrum in which the electrons and holes each form either a spin singlet or triplet. Subsequently, we calculate the oscillator strength, being a measure for the optical transition rates. The spin of the biexciton states relates to two different states of the polarization-entangled photon pair produced in the recombination. We quantify the entanglement of the photon pair depending on the emission directions. The variation of the spectrum and the oscillator strengths due to magnetic or electric fields allows to use ground-state biexcitons in tunnel-coupled QDs as a pulsed source of entangled photon pairs.

We model the biexciton (two electrons and two holes) in two coupled QDs by the Hamiltonian

$$
H=\sum_{\alpha=e, h} \sum_{i=1}^{2} h_{\alpha i}+H_{C}+H_{Z}+H_{E},
$$

where $h_{\alpha i}=\left(\mathbf{p}_{\alpha i}+q_{\alpha} \mathbf{A}\left(\mathbf{r}_{\alpha i}\right) / c\right)^{2} / 2 m_{\alpha}+V_{\alpha}\left(\mathbf{r}_{\alpha i}\right)$ is the single-particle Hamiltonian for the $i$-th electron $(\alpha=e$, $\left.q_{e}=-e\right)$ or hole $\left(\alpha=h, q_{h}=+e\right)$ in two dimensions (2D) with coordinate $\mathbf{r}_{\alpha i}$ and spin $\mathbf{S}^{\alpha i}$. The potential $V_{\alpha}(x, y)=m_{\alpha} \omega_{\alpha}^{2}\left[\left(x^{2}-a^{2}\right)^{2} / 4 a^{2}+y^{2}\right] / 2$ describes two QDs centered at $(x= \pm a, y=0)$, separated by a barrier of height $m_{\alpha} \omega_{\alpha}^{2} a^{2} / 8$. Electrons and holes have effective masses $m_{\alpha}$ and confinement energies $\hbar \omega_{\alpha}$. The Coulomb interaction is included by $H_{C}=$ $(1 / 2) \sum_{(\alpha, i) \neq(\beta, j)} q_{\alpha} q_{\beta} / \kappa\left|\mathbf{r}_{\alpha i}-\mathbf{r}_{\beta j}\right|$, with a dielectric constant $\kappa$ (for bulk GaAs, $\kappa=13.18$ ). A magnetic field $\mathbf{B}$ in $z$ direction leads to orbital effects via the vector potential (in the symmetric gauge) $\mathbf{A}=B(-y, x, 0) / 2$ and to the Zeeman term $H_{Z}=\sum_{\alpha, i} g_{\alpha} \mu_{B} B S_{z}^{\alpha i}$, where $g_{\alpha}$ is the effective g-factor of the electron (hole) and $\mu_{B}$ is the Bohr magneton. Restricting ourselves to the low-energy physics of QDs filled with few particles, we can assume approximately $2 \mathrm{D}$ parabolic confinement. We assume the simultaneous confinement of electrons and holes which can be realized e.g. in QDs formed by thickness fluctuations in a quantum well [8] or by self-assembled QDs [24, 25]. A particle in a single QD is thus described by the Fock-Darwin (FD) Hamiltonian $h_{\alpha}^{ \pm a}\left(\mathbf{r}_{\alpha i}\right)$ [26], comprising a harmonic potential $v_{\alpha}^{ \pm a}(\mathbf{r})=m_{\alpha} \omega_{\alpha}^{2}\left[(x \mp a)^{2}+y^{2}\right] / 2$ and a perpendicular magnetic field. In prospect of the HL ansatz below we write the single-particle part of the Hamiltonian Eq. (11) as $\sum_{\alpha}\left[h_{\alpha}^{-a}\left(\mathbf{r}_{\alpha 1}\right)+h_{\alpha}^{+a}\left(\mathbf{r}_{\alpha 2}\right)\right]+H_{W}\left(\left\{\mathbf{r}_{\alpha i}\right\}\right) \equiv H_{0}+H_{W}$, where $H_{W}\left(\left\{\mathbf{r}_{\alpha i}\right\}\right)=\sum_{\alpha}\left[\sum_{i} V_{\alpha}\left(\mathbf{r}_{\alpha i}\right)-v_{\alpha}^{-a}\left(\mathbf{r}_{\alpha 1}\right)-v_{\alpha}^{+a}\left(\mathbf{r}_{\alpha 2}\right)\right]$. An in-plane electric field $\mathbf{E}=\varepsilon \hat{\mathbf{y}}$ is described by $H_{E}=$ e $\varepsilon\left(y_{e 1}+y_{e 2}-y_{h 1}-y_{h 2}\right)$ and can be included in $H_{0}$. We put $\varepsilon=0$ here and discuss the case $\varepsilon \neq 0$ below.

The valence band is assumed to be split into wellseparated heavy and light hole bands and only heavyhole excitations are considered in the following. The FD ground states $|D\rangle_{\alpha}$ in the $\mathrm{QD} D=1,2$ which are used to make a variational HL ansatz are [26]

$$
\langle\mathbf{r} \mid D\rangle_{\alpha}=\sqrt{\frac{b_{\alpha}}{\pi a_{\alpha}^{2}}} \exp \left(-\frac{b_{\alpha}}{2 a_{\alpha}^{2}}\left((x \pm a)^{2}+y^{2}\right) \pm \frac{i q_{\alpha} a y}{2 e l_{B}^{2}}\right),
$$

where the upper (lower) sign holds for $D=1(2), l_{B}=$ $\sqrt{\hbar c / e B}$ and $b_{\alpha}=\sqrt{1+\left(e B / 2 c m_{\alpha} \omega_{\alpha}\right)^{2}}$.

We now make a strong confinement ansatz by constructing two-particle orbital wave functions for electrons and for holes separately according to the HL method, i.e. a symmetric $\left(|s\rangle^{\alpha} \equiv|I=0\rangle^{\alpha}\right.$, spin singlet) and an antisymmetric $\left(|t\rangle^{\alpha} \equiv|I=1\rangle^{\alpha}\right.$, spin triplet) linear combination of two-particle states $\left|D D^{\prime}\right\rangle_{\alpha}=|D\rangle_{\alpha} \otimes\left|D^{\prime}\right\rangle_{\alpha}$,

$$
|I\rangle^{\alpha}=N_{\alpha I}\left(|12\rangle_{\alpha}+(-1)^{I}|21\rangle_{\alpha}\right),
$$

where $N_{\alpha I}=1 / \sqrt{2\left(1+(-1)^{I}\left|S_{\alpha}\right|^{2}\right)}$ and $S_{\alpha}={ }_{\alpha}\langle 1 \mid 2\rangle_{\alpha}$ denotes the overlap (or tunneling amplitude) between the two orbital wave functions $|1\rangle_{\alpha}$ and $|2\rangle_{\alpha}$. We continue by forming the four biexciton states $|I J\rangle=|I\rangle^{e} \otimes|J\rangle^{h}$, where $I=0$ (1) for the electron singlet (triplet) and $J=0$ (1) for the hole singlet (triplet). The energies

$$
E_{I J}=\langle I J|H| I J\rangle=E^{0}+E^{Z}+E_{I J}^{W}+E_{I J}^{C},
$$

with $E_{I J}^{A} \equiv\left\langle I J\left|H_{A}\right| I J\right\rangle$, can be calculated analytically. In units of $\hbar \omega_{e}$, we find $E_{0} \equiv E_{I J}^{0}=2\left(b_{e}+b_{h} / \eta\right)$, where $\eta=\omega_{e} / \omega_{h}, E^{Z} \equiv E_{I J}^{Z}=\left(\mu_{B} B / \hbar \omega_{e}\right) \sum_{\alpha i} g_{\alpha} S_{z}^{\alpha i}$, and

$$
\begin{aligned}
& E_{I J}^{W}=\frac{3}{16 d^{2}}\left(\frac{1}{b_{e}^{2}}+\frac{\xi}{b_{h}^{2}}\right)-\frac{3 d^{2}}{4}\left(1+\frac{1}{\xi \eta^{2}}\right) \\
& +3 N_{I J}\left[d^{2}\left(1+\frac{1}{\xi \eta^{2}}\right)+(-1)^{J} S_{h}^{2}\left(d^{2}-\frac{1}{\eta b_{h}}\right)\right. \\
& \left.+(-1)^{I} S_{e}^{2}\left(\frac{d^{2}}{\xi \eta^{2}}-\frac{1}{b_{e}}\right)-(-1)^{I+J} S_{e}^{2} S_{h}^{2}\left(\frac{1}{b_{e}}+\frac{1}{\eta b_{h}}\right)\right](5)
\end{aligned}
$$

where $2 d=2 a / a_{e}$ is the dimensionless inter-dot distance, $a_{e}=\sqrt{\hbar / m_{e} \omega_{e}}$ is the electronic Bohr radius, $S_{e}=$ $\exp \left(-d^{2}\left[2 b_{e}-1 / b_{e}\right]\right), S_{h}=\exp \left(-d^{2}\left[2 b_{h}-1 / b_{h}\right] / \xi \eta\right)$, $N_{I J}=N_{e I}^{2} N_{h J}^{2}$, and $\xi=m_{e} / m_{h}$. For $E_{I J}^{\mathrm{C}}$, we find

$$
\begin{aligned}
E_{I J}^{\mathrm{C}}= & \frac{E_{e e}+(-1)^{I} \tilde{E}_{e e}}{1+(-1)^{I} S_{e}^{2}}+\frac{E_{h h}+(-1)^{J} \tilde{E}_{h h}}{1+(-1)^{J} S_{h}^{2}} \\
& +8 N_{I J}\left[E_{X}+E_{e h}+(-1)^{I} S_{e} \tilde{E}_{X e}\right. \\
& \left.+(-1)^{J} S_{h} \tilde{E}_{X h}+(-1)^{I+J} S_{e} S_{h} \tilde{E}_{X e h}\right],
\end{aligned}
$$

where we have used the abbreviations

$$
\begin{aligned}
E_{\alpha \alpha} & =c \sqrt{b_{\alpha} / x_{\alpha}} \exp \left(-b_{\alpha} d^{2} / x_{\alpha}\right) I_{0}\left(b_{\alpha} d^{2} / x_{\alpha}\right) \\
\tilde{E}_{\alpha \alpha} & =c \sqrt{\frac{b_{\alpha}}{x_{\alpha}}} S_{\alpha} \exp \left(-\frac{b_{\alpha} d^{2}}{x_{\alpha}}\right) I_{0}\left(\frac{d^{2}}{x_{\alpha}}\left[b_{\alpha}-\frac{1}{b_{\alpha}}\right]\right) \\
E_{X} & =-c \sqrt{\bar{b}} \\
E_{e h} & =E_{X} \exp \left(-\bar{b} d^{2}\right) I_{0}\left(\bar{b} d^{2}\right) \\
\tilde{E}_{X \alpha} & =2 S_{\alpha} E_{X} \exp \left(-\bar{b} d^{2} / 4 b_{\alpha}^{2}\right) I_{0}\left(\bar{b} d^{2} / 4 b_{\alpha}^{2}\right) \\
\tilde{E}_{X e h} & =S_{e} S_{h} E_{X}\left\{\exp \left(\bar{b}_{1} d^{2} / 2\right) I_{0}\left(\bar{b}_{1} d^{2} / 2\right)\right. \\
& \left.\quad+\exp \left(\bar{b}_{2} d^{2} / 2\right) I_{0}\left(\bar{b}_{2} d^{2} / 2\right)\right\}
\end{aligned}
$$

Here, $I_{0}(x)$ is the zeroth-order modified Bessel function, $c=e^{2} \sqrt{\pi / 2} / \kappa a_{e} \hbar \omega_{e}$ is a dimensionless parameter characterizing the Coulomb interaction, $x_{e}=1, x_{h}=\xi \eta$, 
$\bar{b}=2 b_{e} b_{h} /\left(b_{h}+\xi \eta b_{e}\right), \bar{b}_{1}=b_{e}-1 / b_{e}+\left[b_{h}-1 / b_{h}\right] / \xi \eta$, and $\bar{b}_{2}=\left(\left[b_{e}-1 / b_{e}\right]\left[b_{h}-2 \xi \eta b_{e}\right]+b_{e}\left[b_{h}-1 / b_{h}\right]\right) /\left(b_{h}+\xi \eta b_{e}\right)$. Fig. 11 shows the biexciton energies $E_{I J}(I, J=0,1=s, t)$ in the double QD as a function of an applied external magnetic field in $z$ direction. The Zeeman interaction $H_{Z}$ causes an additional level splitting of $\approx 0.02 \hbar \omega_{e} / \mathrm{T}$ (assuming $\left|g_{e}\right| \approx\left|g_{h}\right| \approx 1$ ) for the triplet states with $\sum_{i} S_{z}^{\alpha i} \neq 0$ which is not shown in Fig. 1. The electronhole exchange interaction for the GaAs QDs considered here is reported to be only on the order of tens of $\mu \mathrm{eV}$ [27] and can therefore be neglected. The self-consistency of omitting excited single-QD states in the HL ansatz can be checked by comparing the energy $E_{I J}^{C}+E_{I J}^{W}$ to the single-QD level spacing. This criterion is fulfilled for inter-dot distances $2 a \gtrsim 20 \mathrm{~nm}$. In addition to the HL states $|I J\rangle$, we consider the double occupation states $|D D D D\rangle$ for which all four particles are located on the same QD $D=1,2$. Their energies are given by $\bar{E}=$ $E^{0}+E^{Z}+\bar{E}^{W}+\bar{E}^{C}$, with $\bar{E}^{W}=3\left(1 / b_{e}^{2}+\xi / b_{h}^{2}\right) / 16 d^{2}$, and $\bar{E}^{C}=c\left(\sqrt{b_{e}}+\sqrt{b_{h} / \xi \eta}-4 \sqrt{\bar{b}}\right)$.

We proceed to the calculation of the oscillator strengths of biexciton-exciton and exciton-vacuum transitions. The oscillator strength $f$ is a measure for the coupling of exciton states to the electromagnetic field and is proportional to the optical transition rates. For a transition between the $N+1$ and $N$ exciton states $|N+1\rangle$ and $|N\rangle$, the oscillator strength is defined as

$$
f_{N+1, N}=2\left|p_{N \mathbf{k} \lambda}\right|^{2} / m_{0} \hbar \omega_{N+1, N}
$$

where $m_{0}$ is the bare electron mass, $\hbar \omega_{N+1, N}=E_{N+1}-$ $E_{N}$, and $p_{N \mathbf{k} \lambda}=\left\langle N+1\left|\mathbf{e}_{\mathbf{k} \lambda} \cdot \mathbf{p}\right| N\right\rangle$, where $\mathbf{e}_{\mathbf{k} \lambda}$ is the unit polarization vector for a photon with momentum $\mathbf{k}$ and helicity $\lambda= \pm 1$, and $\mathbf{p}$ is the electron momentum operator. For $p_{N \mathbf{k} \lambda}$ we find in the dipole approximation
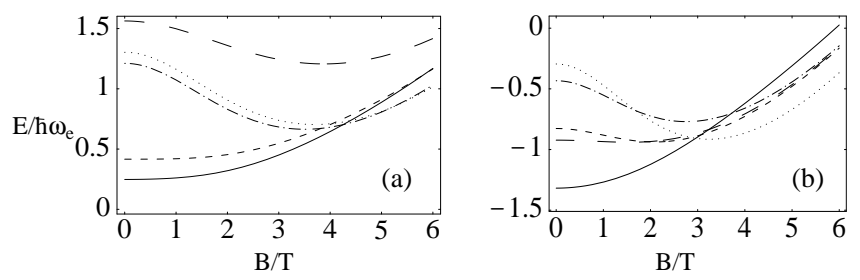

FIG. 1: Biexciton energies in units of $\hbar \omega_{e}$ for (a) $\eta=\omega_{e} / \omega_{h}=$ $1 / 2$, (b) $\eta=1 / \xi=1.67\left(a_{e}=a_{h}\right)$, in a $2 \mathrm{D}$ GaAs system $\left(m_{e}=0.067 m_{0}, m_{h h}=0.112 m_{0}\right), \hbar \omega_{e}=3 \mathrm{meV}$, and $d=0.7$. The plotted HL energies $E_{I J}$ are $E_{s s}$ (solid line), $E_{s t}$ (shortdashed), $E_{t s}$ (dot-dashed), and $E_{t t}$ (dotted), neglecting the Zeeman energy. The exchange splittings $E_{t J}-E_{s J}, J=s, t$, for electrons are larger than for holes $\left(E_{I t}-E_{I s}, I=s, t\right)$ in (a) where $\eta \xi<1$, but of the same order in (b) $(\eta \xi=1)$. At $B=0,|s s\rangle$ has the lowest energy, while for larger $B$, there is a crossover to a $|t t\rangle$ ground state. Double occupation of a QD (long-dashed line) becomes more favorable with increasing $\eta$; in (a), $\bar{E}>E_{I J}, I, J=s, t$, while in (b), $\bar{E}$ is smaller than some of the $E_{I J}$ for small $B$. $a_{\alpha} \ll 2 \pi / k\left(a_{\alpha} \approx 20 \mathrm{~nm}, 2 \pi / k \approx 1 \mu \mathrm{m}\right)$,

$$
\begin{aligned}
& p_{N \mathbf{k} \lambda}=[(N+1) !]^{2} \sum_{\left\{\sigma_{i}, \tau_{j}\right\}, \sigma} M_{\sigma \lambda}(\theta) \int d^{3} r \prod_{i, j} d^{3} r_{i} d^{3} s_{j} \\
& \left.\times \Phi_{N}\left(\left\{\mathbf{r}_{i}, \sigma_{i}\right\} ;\left\{\mathbf{s}_{j}, \tau_{j}\right\}\right) \Phi_{N+1}^{*}\left(\left\{\mathbf{r}_{i}, \sigma_{i}\right\}, \mathbf{r}, \sigma ;\left\{\mathbf{s}_{j}, \tau_{j}\right\}, \mathbf{r}, \sigma\right\}\right),
\end{aligned}
$$

where $\Phi_{N}$ is the $N$-exciton wavefunction, depending on the conduction-band electron (valence-band hole) coordinates $\mathbf{r}_{i}\left(\mathbf{s}_{j}\right)$ and their spins $\sigma_{i}\left(\tau_{j}\right)(i, j=1 \ldots N)$. The coordinate and spin of the electron and the hole created or annihilated during the optical transition are denoted by $\mathbf{r}$ and $\sigma$. The inter-band momentum matrix element for a cubic crystal symmetry is given by $M_{\sigma \lambda}(\theta)=\mathbf{e}_{\mathbf{k} \lambda} \cdot \mathbf{p}_{c v}(\sigma)=p_{c v}(\cos (\theta)-\sigma \lambda) / 2 \equiv p_{c v} m_{\sigma \lambda}(\theta)$, where $\theta$ is the angle between $\mathbf{k}$ and the normal to the plane of the 2D electron system (assuming that the latter coincides with one of the main axes of the cubic crystal), and $E_{p}=2 p_{c v}^{2} / m_{0}(=25.7 \mathrm{eV}$ for GaAs $)$.

According to Eq. (14), the orbital momentum matrix element for transitions from the exciton vacuum $|0\rangle$ to an exciton state $|X\rangle=|D\rangle_{e} \otimes|D\rangle_{h} \equiv|D D\rangle$ in one $\mathrm{QD}$ (or for the optical recombination of $|X\rangle$ ) is $p_{0}=M_{\sigma \lambda}(\theta) \int d^{3} r \Phi_{1}^{*}(\mathbf{r}, \mathbf{r}) \equiv M_{\sigma \lambda}(\theta) C_{e h}$. The exciton wave function is denoted by $\Phi_{1}\left(\mathbf{r}_{e}, \mathbf{r}_{h}\right)=\left\langle\mathbf{r}_{e}, \mathbf{r}_{h} \mid X\right\rangle$. From this, we find for the oscillator strength

$$
f_{X, 0}=\frac{2\left|p_{0}\right|^{2}}{m_{0} \hbar \omega_{X, 0}}=\frac{E_{p}}{\hbar \omega_{X, 0}} M_{\sigma \lambda}(\theta)^{2}\left|C_{e h}\right|^{2},
$$

and $C_{e h}=2 \sqrt{\xi \eta b_{e} b_{h}} /\left(b_{h}+\xi \eta b_{e}\right)$. In Fig. 2a we plot $\left|C_{e h}\right|^{2}=f / f_{0}$ as a function of the magnetic field, where $f_{0}=E_{p} m_{\sigma \lambda}(\theta)^{2} / E_{g}$ denotes the oscillator strength for (bulk) inter-band transitions, equating $\hbar \omega_{X, 0}$ with the band-gap energy $E_{g}$. Since we have made a strong confinement ansatz, the obtained oscillator strength is independent of the QD volume $V$. For weak confinement, one would expect $f \propto V$. Fig. $2 \mathrm{~b}$ shows the suppression of the exciton transition rate by an electric field.

The momentum matrix element $p_{1}$ for transitions from an exciton state $|X\rangle$ to a biexciton state $|X X\rangle$ is given by $p_{1}=-2 M_{\sigma \lambda}(\theta) \int d^{3} r_{e} d^{3} r_{h} d^{3} r \Phi_{2}^{*}\left(\mathbf{r}_{e}, \mathbf{r} ; \mathbf{r}_{h}, \mathbf{r}\right) \Phi_{1}\left(\mathbf{r}_{e}, \mathbf{r}_{h}\right)$. If the recombining electron and hole are on the same $\mathrm{QD}$, the integral over $\mathbf{r}$ yields $C_{e h}$, otherwise $S_{e h}=$ $C_{e h} \exp \left(-2 d^{2}\left[b_{e}-\xi \eta /\left(b_{h}+\xi \eta b_{e}\right)\right]\right)$.
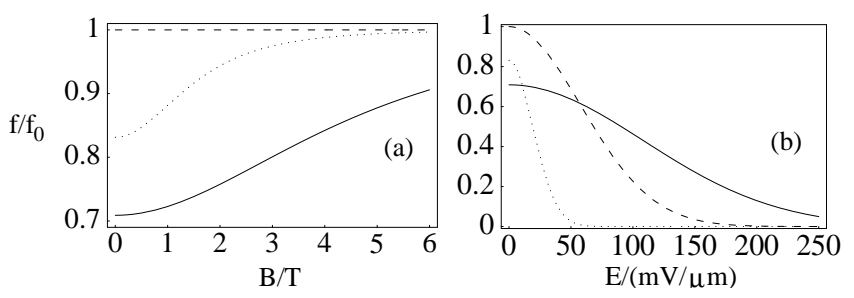

FIG. 2: Oscillator strength $f_{X, 0}$ for GaAs QDs in units of $f_{0}$ as a function of (a) the magnetic field $B$ (in Tesla) at $E=0$ and (b) the electric field $E$ (in $\mathrm{mV} / \mu \mathrm{m}$ ) at $B=0$, with $\eta=\omega_{e} / \omega_{h}=1 / 2$ (solid line), $\eta=1 / \xi$ (dashed), $\eta=4$ (dotted). For $\eta=1 / \xi$ the $B$ field has no effect on $C_{e h}$. 
We give here our result for $p_{1}$ for a transition between the HL biexciton states $|X X\rangle=|I J\rangle$ with one exciton on each QD and a single exciton in the final state $|X\rangle=$ $|D D\rangle$, a single exciton on $\operatorname{dot} D=1,2$,

$$
\begin{aligned}
& \left|\left\langle I J\left|\mathbf{e}_{\mathbf{k} \lambda} \cdot \mathbf{p}\right| D D\right\rangle\right|=2 M_{\sigma \lambda}(\theta) \sqrt{N_{I J}} \\
& \times\left(C_{e h}\left[(-1)^{I+J}+S_{e} S_{h}\right]+S_{e h}\left[(-1)^{J} S_{e}+(-1)^{I} S_{h}\right] \times 16\right)
\end{aligned}
$$

Approximating $\hbar \omega_{X X, X} \approx E_{g}$, we plot the corresponding oscillator strength versus $B$ and $E$ in Figs. 3 a and 3 b.

Results for $f_{X X, X}$, also including the (biexciton) double occupation state $|D D D D\rangle$ and various final (exciton) states, will be given elsewhere [28].
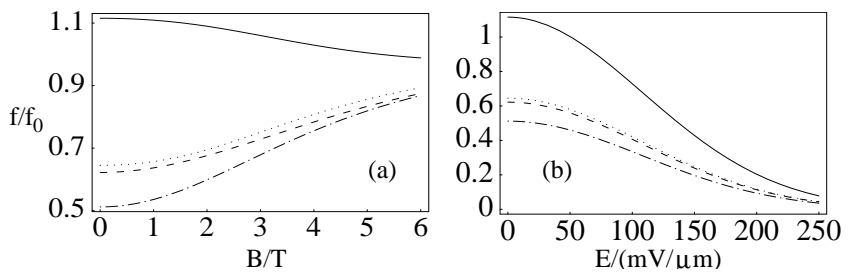

FIG. 3: Oscillator strengths $f_{X X, X}$ for transitions between the biexciton states $|X X\rangle=|I J\rangle$ and a single remaining exciton on one QD in units of $f_{0}$ as a function of (a) the magnetic field $B$ (in Tesla) at $E=0$ and (b) the electric field $E$ (in $\mathrm{mV} / \mu \mathrm{m})$ at $B=0$. The parameters were chosen for GaAs with $\eta=\omega_{e} / \omega_{h}=1 / 2$. The line styles correspond to those for $E_{I J}$ in Fig. 11.

The main effect of an electric field is to spatially separate the electrons from the holes [29], which leads to a reduction of the oscillator strengths [28] (cf. Figs. 2b and $3 \mathrm{~b}$ ). Hence, the optical transition rate can be efficiently switched off and on, thus allowing the deterministic emission of one photon pair.

Transformation of a HL biexciton state $|I J\rangle$ into the basis of two coupled excitons yields a superposition of dark $\left(S_{z}= \pm 2\right)$ and bright $\left(S_{z}= \pm 1\right)$ exciton states.
The emitted photon states are (up to normalization)

$$
\left|\chi_{I J}\right\rangle \propto\left|+1, \theta_{1}\right\rangle\left|-1, \theta_{2}\right\rangle+(-1)^{I+J}\left|-1, \theta_{1}\right\rangle\left|+1, \theta_{2}\right\rangle,
$$

where $|\sigma, \theta\rangle=N(\theta)\left(m_{\sigma,+1}(\theta)\left|\sigma_{+}\right\rangle+m_{\sigma,-1}(\theta)\left|\sigma_{-}\right\rangle\right)$is the state of a photon emitted from the recombination of an electron with spin $S_{z}=\sigma / 2= \pm 1 / 2$ and a heavy hole with spin $S_{z}=3 \sigma / 2$ in a direction which encloses the angle $\theta$ with the normal to the plane of the $2 \mathrm{D}$ electron and hole motion. The states of right and left circular polarization are denoted $\left|\sigma_{ \pm}\right\rangle$.

The entanglement of the state (17) can be quantified by the von Neumann entropy $E$. For $|s s\rangle$ or $|t t\rangle$ and emission of the two photons enclosing an azimuthal angle $\phi=0$ or $\pi$, we obtain $E=\log _{2}\left(1+x_{1} x_{2}\right)-x_{1} x_{2} \log _{2}\left(x_{1} x_{2}\right) /(1+$ $\left.x_{1} x_{2}\right)$, where $x_{i}=\cos ^{2}\left(\theta_{i}\right)$. Note that only the emission of both photons perpendicular to the plane $\left(\theta_{1}=\theta_{2}=0\right)$ results in maximal entanglement $(E=1)$ since only in this case $\left|+1, \theta_{i}\right\rangle$ is orthogonal to $\left|-1, \theta_{i}\right\rangle$. In particular, the two photons are not entangled $(E=0)$ if at least one of them is emitted in-plane $\left(\theta_{i}=\pi / 2\right)$. To observe the proposed effect, the relaxation rate to the biexciton ground state must exceed the biexciton recombination rate. That such a regime can be reached is suggested by experiments with low excitation densities, see e.g. [30]. Then, an upper limit for the pair production rate is given by $\left(\tau_{X}+\tau_{X X}\right)^{-1}$, where $\tau_{X, X X}$ is the (bi)exciton lifetime.

Conversely, spin-entangled electrons can be produced by optical absorption followed by relaxation of the biexciton to its ground state. After each QD has been filled with an exciton, the recombination can be suppressed by an electric field. Having removed the holes, the electron singlet and triplet could then in principle be distinguished by a subsequent interference experiment 31.

We thank A. V. Khaetskii, A. Imamoḡlu, and P. Petroff for discussions. We acknowledge support from the Swiss NSF, DARPA, and ARO.
[1] C.H. Bennett, D.P. DiVincenzo, Nature 404, 247 (2000).

[2] D. Loss, D. P. DiVincenzo, Phys. Rev. A 57, 120 (1998).

[3] P. Recher, E. V. Sukhorukov, D. Loss, Phys. Rev. B 63, 165314 (2001).

[4] O. Benson et al., Phys. Rev. Lett. 84, 2513 (2000).

[5] E. Moreau et al., Phys. Rev. Lett. 87, 183601 (2001).

[6] F. Troiani, U. Hohenester, E. Molinari, Phys. Rev. B 62, R2263 (2000);

[7] P. Chen, C. Piermarocchi, L. J. Sham, Phys. Rev. Lett. 87, 067401 (2001).

[8] G. Chen et al., Science 289, 1906 (2000).

[9] L. Quiroga, N. F. Johnson, Phys. Rev. Lett. 83, 2270 (1999).

[10] M. Bayer et al., Science 291, 451 (2001).

[11] E. Biolatti et al., Phys. Rev. B 65, 075306 (2002).

[12] Al. L. Efros, A. L. Efros, Sov. Phys. Semicond. 16, 772 (1982).
[13] L. Banyai et al., Phys. Rev. B 38, 8142 (1988).

[14] T. Takagahara, Phys. Rev. B 39, 10206 (1989).

[15] G. W. Bryant, Phys. Rev. B 41, 1243 (1990).

[16] Y. Z. Hu et al., Phys. Rev. Lett. 64, 1805 (1990); Phys. Rev. B 42, 1713 (1990).

[17] S. V. Nair, T. Takagahara, Phys. Rev. B 55, 5153 (1996).

[18] P. Hawrylak, Phys. Rev. B 60, 5597 (1999).

[19] A. Kiraz et al., cond-mat/0108450.

[20] C. Santori et al., cond-mat/0108466.

[21] G. Schedelbeck et al., Science 278, 1792 (1997).

[22] E. Johnston-Halperin et al., Phys. Rev. B 63, 205309 (2001).

[23] W. F. Brinkman, T. M. Rice, B. Bell, Phys. Rev. B 8, 1570 (1973).

[24] R. J. Luyken et al., Physica E 2, 704 (1998).

[25] T. Lundstrom et al., Science 286, 2312 (1999).

[26] G. Burkard, D. Loss, D. P. DiVincenzo, Phys. Rev. B 59, 
2070 (1999).

[27] D. Gammon et al., Science 273, 87 (1996).

[28] O. Gywat, G. Burkard, D. Loss, to be published.

[29] For non-identical dots, electric fields can also be used to tune the lowest electron levels into resonance which is sufficient for the generation of entangled photons or electrons.

[30] B. Ohnesorge et al., Phys. Rev. B 54, 11532 (1996).

[31] G. Burkard, D. Loss, E. V. Sukhorukov, Phys. Rev. B 61, R16303 (2000). 